\title{
The Effect of Oxygen Uptake on Charge Injection Barriers in Conjugated Polymer Films
}

Qinye Bao, Xianjie Liu, Slawomir Braun, Jianming Yang, Yanqing Li, J ianxin Tang, Chungang Duan and Mats Fahlman

The self-archived postprint version of this journal article is available at Linköping University Institutional Repository (DiVA):

http:// urn.kb.se/ resolve?urn=urn:nbn:se:liu:diva- 145757

N.B.: When citing this work, cite the original publication.

Bao, Q., Liu, X., Braun, S., Yang, J., Li, Y., Tang, J., Duan, C., Fahlman, M., (2018), The Effect of Oxygen Uptake on Charge Injection Barriers in Conjugated Polymer Films, ACS Applied Materials and Interfaces, 10(7), 6491-6497. https:// doi.org/ 10.1021/acsami.7b17368

Original publication available at:

https:// doi.org/ 10.1021/acsami.7b17368

Copyright: American Chemical Society

http:// pubs.acs.org/ 


\title{
Article
}

\section{The Effect of Oxygen Uptake on Charge Injection Barriers in Conjugated Polymer Films}

Qinye Bao ${ }^{1,2,3^{*}}$, Xianjie $\mathrm{Liu}^{2}$, Slawomir Braun${ }^{2}$, Jianming Yang ${ }^{1}$, Yanqing $\mathrm{Li}^{4}$, Jianxin Tang $^{4}$, Chungang Duan ${ }^{1}$, Mats Fahlman ${ }^{2}$

1 Key Laboratory of Polar Materials and Devices, Ministry of Education, East China Normal University, 200241, Shanghai, P.R. China

*E-mail: qybao@clpm.ecnu.edu.cn

2 Division of Surface Physics and Chemistry, IFM, Linköping University SE-58183 Linköping, Sweden

3 Jiangsu Key Laboratory for Carbon-Based Functional Materials \& Devices, Soochow University, Suzhou 215123, P. R. China

4 Institute of Functional Nano \& Soft Materials (FUNSOM), Soochow University, Suzhou 215123, P. R. China

Keywords: Charge injection; barrier; spectroscopy; conjugated polymer; organic electronics

\begin{abstract}
:
The energy offset between the electrode Fermi level and organic semiconductor transport levels is a key parameter controlling the charge injection barrier and hence efficiency of organic electronic devices. Here, we systematically explore the effect of in situ oxygen exposure on energetics in n-type conjugated polymer P(NDI2OD-T2) films. The analysis reveals that an interfacial potential step is introduced for a series of P(NDI2OD-T2)-electrode contacts, causing a nearly constant downshift of the vacuum level while the ionization energies versus vacuum level remain constant. These findings are attributed to the establishment of a so-called double dipole step via motion of charged molecules and will modify the charge injection barriers at electrode contact. We further demonstrate that the same behavior occurs when oxygen interacts with ptype polymer TQ1 films, indicating it is possible to be a universal effect for organic
\end{abstract}


semiconductors.

\section{Introduction}

Conjugated polymers exhibit optical and electronic properties which make them suitable for organic electronics applications, e.g., thin film transistors, ${ }^{1-2}$ light emitting diodes, ${ }^{3-4}$ and photovoltaic cells. ${ }^{5-6}$ The electronic structure at heterojunctions often plays a critical role in the performance and functionality of such devices, governing e.g. charge injection/collection. ${ }^{7-9}$ Many conjugated polymers unfortunately are sensitive to ambient atmosphere, in particular to oxygen exposure, leading to changes in electronic structure. These changes, involving oxidization/doping of the semiconducting polymers, typically are accelerated by light exposure and elevated temperatures. ${ }^{10-12}$ Previous work by Nicolai, et al, demonstrated that organic semiconductor films feature electron trap states at $\sim 3.6 \mathrm{eV}$ below vacuum level. These trap states, which have a significant effect on the charge transport, are suggested to originate in bishydrated-oxygen complexes created by water and oxygen uptake. ${ }^{13}$ Light-induced p-type doping also can occur, as demonstrated for P3HT exposed to oxygen by Hintz, et al, a process found to be reversible by vacuum annealing. ${ }^{14}$ Unless the polymers films are protected from exposure to ambient atmosphere, the effects of p-doping and electron trap state formation appear in organic photovoltaic cells (OPVs) resulting in decreased shortcircuit current, open circuit voltage, fill factor, and sometimes even by the formation of an "S"-shaped current-voltage curve. ${ }^{15}$ In organic field-effect transistors (OFETs) such processes show up as a shift in threshold voltage, reduction in mobility, decreased 
subthreshold slope, and increased off-current. ${ }^{16}$ Hence, understanding the effects on (interface) energetics caused by oxygen in organic semiconductor films certainly is an essential step for future development of more robust organic materials and devices.

Poly\{[N,N'-bis(2-octyldodecyl)-naphthalene-1,4,5,8-bis(dicarboximide)-2,6-diyl]-alt-5,5'-(2,2' bithiophene) (P(NDI2OD-T2), the chemical structure of which is depicted in Fig. 1a, is widely used in OPVs and OFETs as a n-type polymer semiconductor thanks to the red-shifted optical absorption relative to the fullerene derivatives and excellent electron mobility, as high as $0.85 \mathrm{~cm}^{2} \mathrm{~V}^{-1} \mathrm{~S}^{-1} \cdot{ }^{17-18}$ Charge accumulation spectroscopy and Raman spectroscopy have been used to study the degradation phenomena of $\mathrm{P}(\mathrm{NDI} 2 \mathrm{OD}-\mathrm{T} 2)$ films exposed to oxygen, where electron trapping resulting in decreased charge transport ability was found to occur, and the electron traps were induced by an $\mathrm{O}_{2} \bullet \bullet P(N D I 2 O D-T 2)$ complex formation involving slight destabilization of the polymer highest occupied molecular orbital (HOMO) and creation of an inter-gap level localized mainly on the $\mathrm{O}_{2}$ molecule, and the comparatively weak oxygen-polymer interaction was suggested to be reversible by heating. ${ }^{19}$ In this paper, we investigate the influence of oxygen on P(NDI2OD-T2) film interface energetics by controlled in situ oxygen gas exposure probed by ultraviolet and X-ray photoelectron spectroscopy (UPS and XPS). We address the evolution in work function, the frontier feature (HOMO) and ionization potential (IP) by UPS so as to monitor the interface energetics and hence injection barriers during the exposure. XPS is applied to probe whether direct bond formation between oxygen and P(NDI2OD-T2) occurs or is indeed 
weak as previously suggested by calculations. ${ }^{19}$ To exclude the effects (e.g. photooxidation) by other sources like sun light, especially the UV part, the sample is shielded and the experiments are carried out without exposure to UV-Vis light. Our in situ approach enables us to track the oxygen-induced charge injection barrier modifications between electrode Fermi level and organic semiconductor energy level, and discuss their origins.

\section{Results and Discussion}

a
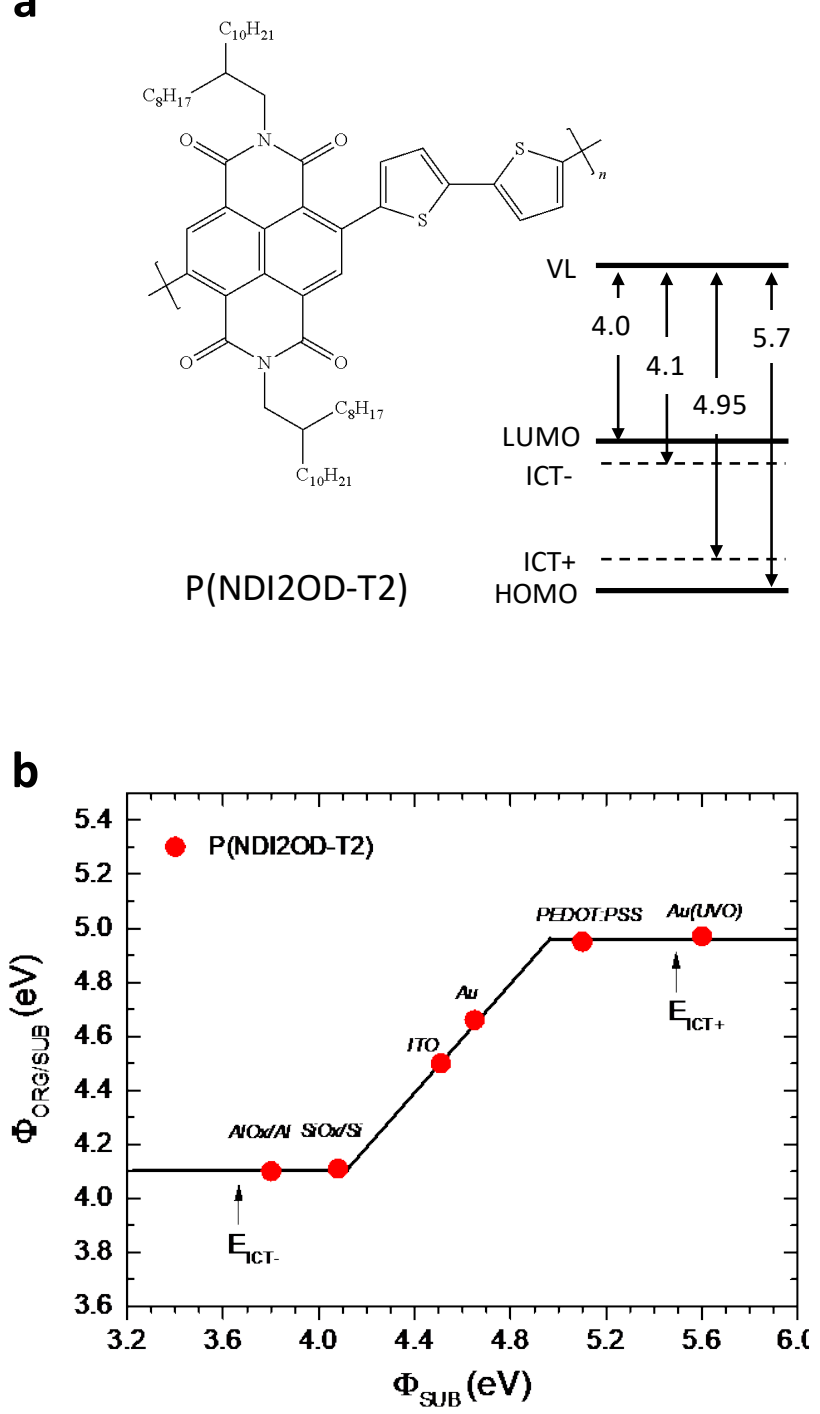

Figure 1. (a). Chemical structure of n-type conjugated polymer P(NDI2OD-T2) with a diagram of the energy level. ICT+ and ICT- represent the energy level of positive and negative integer charge transfer state, respectively; (b) 
Energetics at P(NDI2OD-T2) interface that follows the ICT model: dependences of the work function of P(NDI2OD-

T2) film coated various conducting substrates via solution process, $\Phi_{\mathrm{ORG} / \mathrm{SUB}}$ on the work function of bare substrate, $\Phi_{\text {suB. }}$

The energetics at weakly-interacting organic/electrode (organic) interface follow the integer charge transfer (ICT) model, which describes the relation between the Fermi level and the integer charge transfer states of the organic semiconductor (see supporting information). ${ }^{7,20-24}$ The Fermi level pinning results from spontaneous charge transfer across the interface when there is an energy difference between the substrate work function and the energy required to oxide/ gained from reducing a molecule at the interface. ${ }^{7,25}$ Fig. $1 \mathrm{~b}$ shows the energetics at P(NDI2OD-T2) interface that follows the typical ICT model, where the P(NDI2OD-T2) films were deposited onto various substrates with a broad range of work function to cover all three distinct regimes. The measured $\mathrm{E}_{\mathrm{ICT}+,-}$ of the pristine P(NDI2OD-T2) film is determined to be 4.95 and 4.1 $\mathrm{eV}$, respectively. When the underlying substrate work function $\Phi_{\text {suB }}$ is larger (smaller) than $4.95 \mathrm{eV}(4.1 \mathrm{eV})$, the Fermi level is pinned to $\mathrm{E}_{\text {ICT+ }}\left(\mathrm{E}_{\text {ICT- }}\right)$ with the formation of the downshift (upshift) potential step at interface, the resulting work function of the film $\Phi_{\mathrm{ORG} / \mathrm{SUB}}$ is constant with the value of $\mathrm{E}_{\text {ICT+ }}$ ( $\mathrm{E}_{\text {ICT- }}$ ). In the vacuum level alignment region $\left(4.1 \mathrm{eV}<\Phi_{\mathrm{SUB}}<4.95 \mathrm{eV}\right)$, the $\Phi_{\mathrm{ORG} / \mathrm{SUB}}$ of the $\mathrm{P}(\mathrm{NDI} 2 \mathrm{OD}-\mathrm{T} 2)$ film is equal to $\Phi_{\text {suB }}$ which is the case for the P(NDI2OD-T2) films coated on indium tin oxide (ITO, $\left.\Phi_{\mathrm{SUB}}=4.5 \mathrm{eV}\right)$ and gold $\left(\mathrm{Au}, \Phi_{\mathrm{SUB}}=4.7 \mathrm{eV}\right)$. 
a

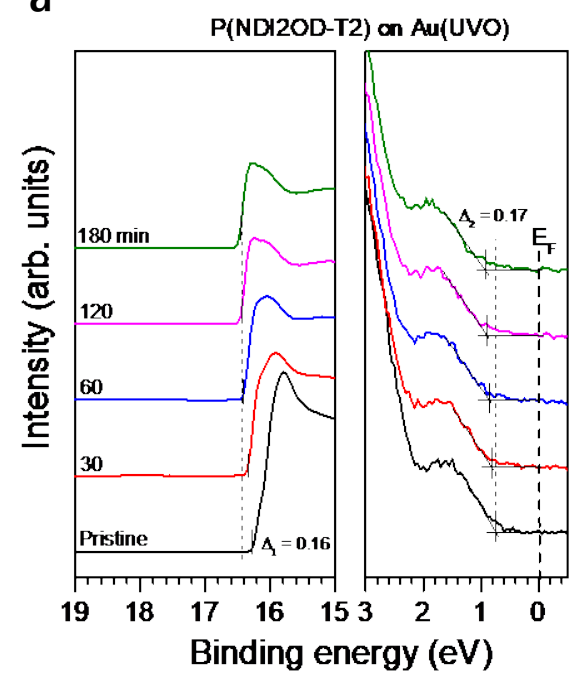

C

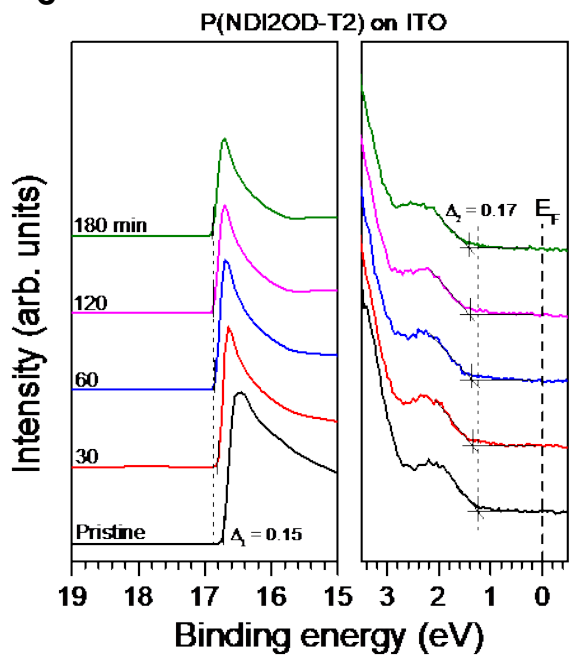

e

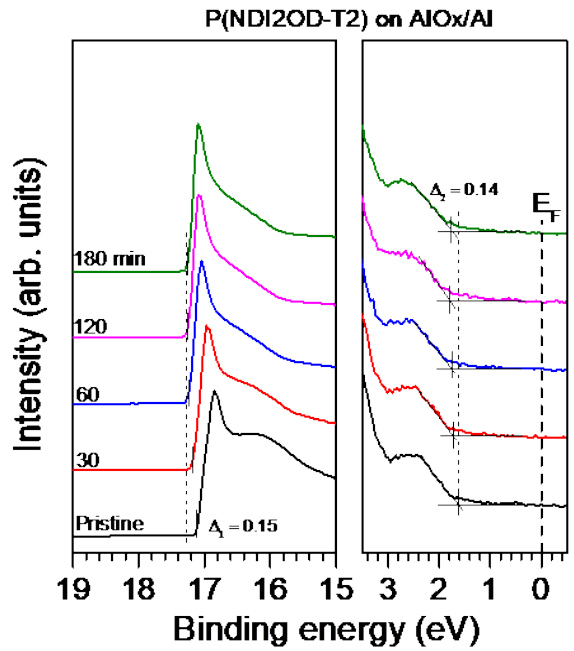

b
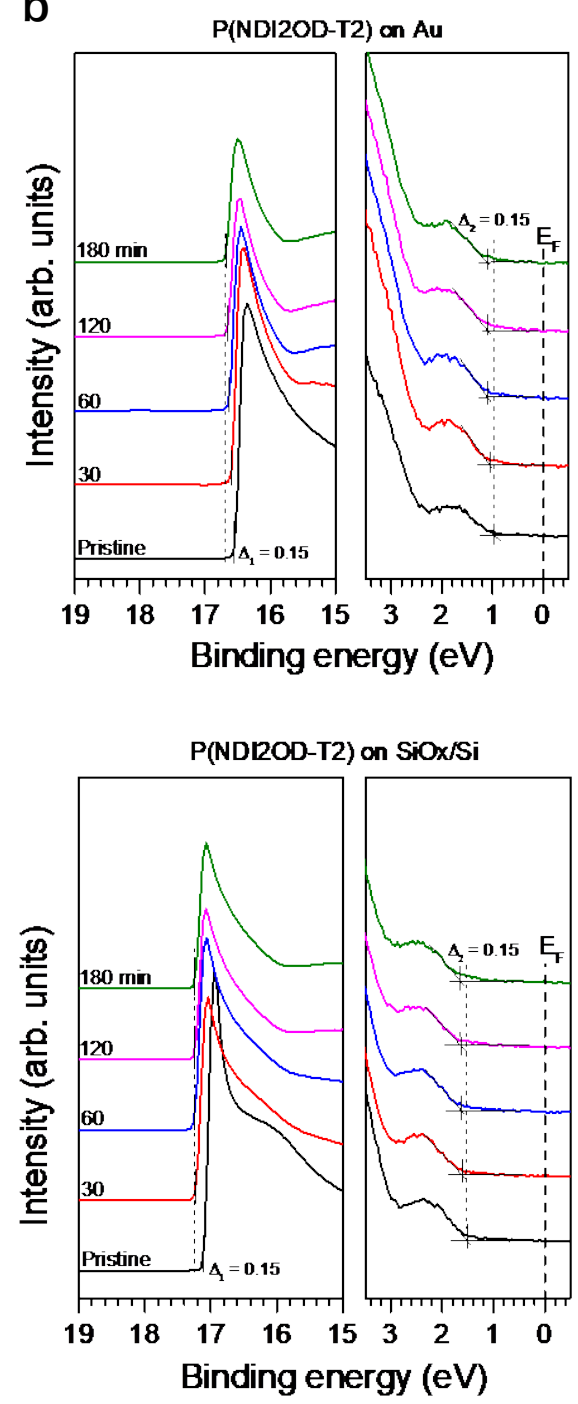

f

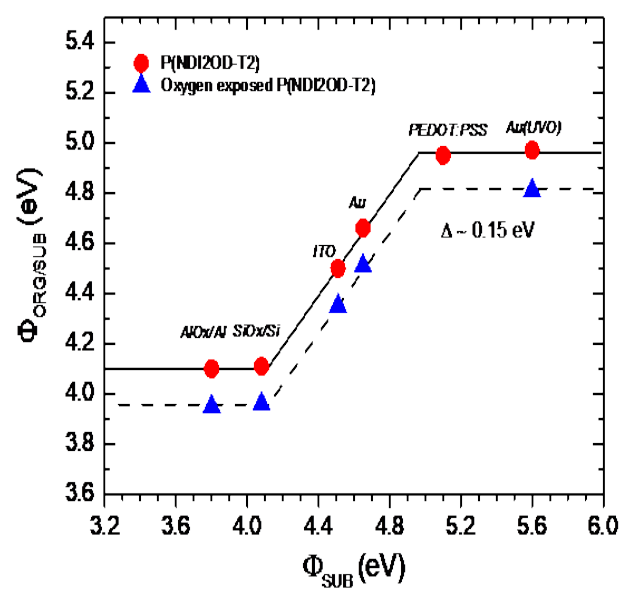

Figure 2. (a)-(e) UPS spectra evolution at the secondary electron region (work function, left) and the frontier electronic structure region (HOMO, right) of P(NDI2OD-T2) film on various conducting substrates including $\mathrm{Au}(\mathrm{UVO}), \mathrm{Au}, \mathrm{ITO}, \mathrm{SiOx} / \mathrm{Si}$ and $\mathrm{AlOx} / \mathrm{Al}$, as a function of in situ oxygen gas exposure time 30, 60, 120 and 180 
min. The bottom spectrum corresponds to the pristine film for comparison. $\Delta 1$ and $\Delta 2$ are contributed to the energy shift of work function and HOMO edge with respect to $E_{F}$, respectively. (f) Summary of the resulting ICT plots of $\mathrm{P}(\mathrm{NDI} 2 \mathrm{OD}-\mathrm{T} 2)$ after saturated oxygen exposure. The energy shift of about $0.15 \mathrm{eV}$ under the original ICT curve is shown.

Fig. 2a-e displays the UPS spectra evolutions of the P(NDI2OD-T2) films on conducting substrates covering the all three regimes of the ICT model, as a function of exposure time under controlled oxygen gas pressure. In Fig. 2a the bottom spectrum corresponds to the pristine P(NDI2OD-T2) film on UV-ozone treated Au (Au UVO) substrate, and its work function and the IP are estimated from the results to be 4.95 and $5.6 \mathrm{eV}$, respectively. Since the work function of the Au UVO substrate is $5.6 \mathrm{eV}$, it causes the formation of occupied ICT+ states in the P(NDI2OD-T2) film at the interface, pining the work function to the $\mathrm{E}_{\mathrm{ICT}+}$, as visible in Fig. 1b. For oxygen exposure time from 30 to $180 \mathrm{~min}$, the vacuum level shifts downward by $0.15 \mathrm{eV}$ and remains stable. Synchronously, the HOMO edge gradually shifts to higher binding energy by $0.15 \mathrm{eV}$. There are neither changes in the IP nor in the spectral features of P(NDI2OD-T2) film. The same behavior exhibited in Fig. 2b-e, occurs for both the case of pinning to ICTstates [P(NDI2OD-T2) on $\mathrm{SiOx} / \mathrm{Si}$ and $\mathrm{AlOx} / \mathrm{Al}$, respectively] and the case of the vacuum level alignment [P(NDI2OD-T2) on Au and ITO, respectively]. From the results presented in Fig. 2f, all the work functions of the P(NDI2OD-T2) films after exposure to oxygen gas shift by $0.15 \mathrm{eV}$ below the original ICT curve. 

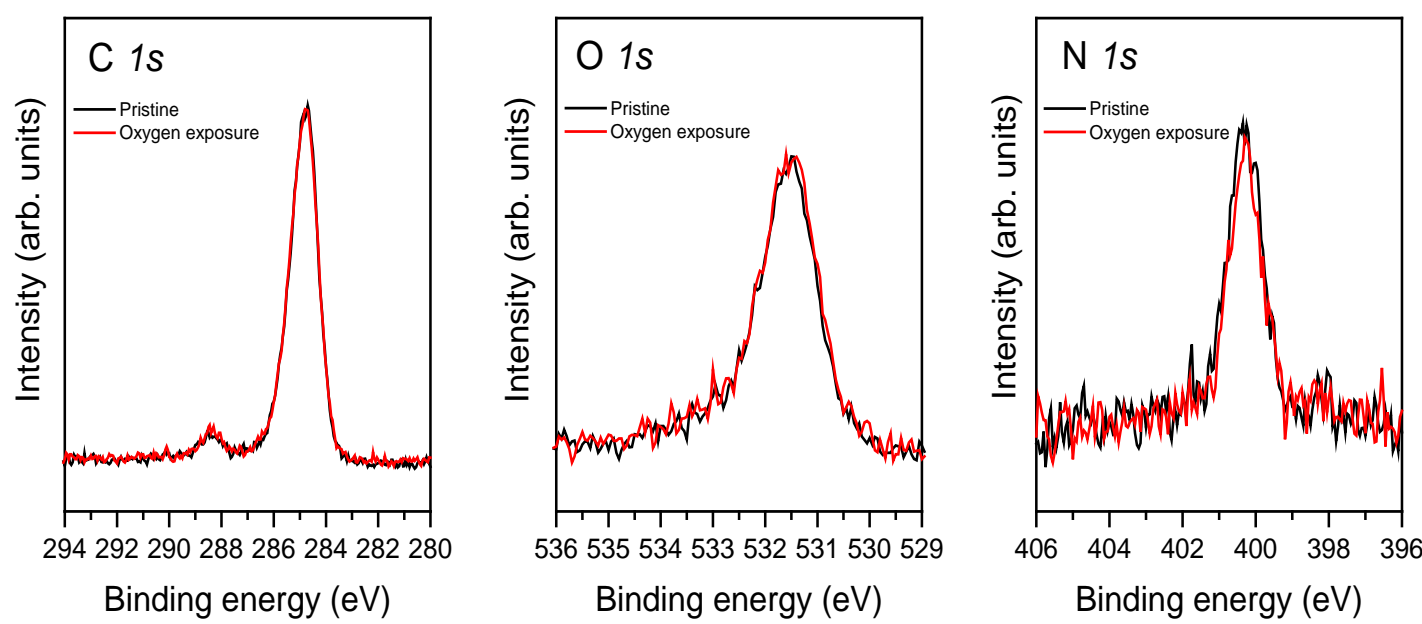

Figure 3. XPS core level spectra C1s, O1s and N1s of P(NDI2OD-T2) film before and after 180 min oxygen gas exposure.

The findings are similar to recent results on other organic semiconductors, e.g. PCBM interface upon oxygen exposure, ${ }^{25}$ where it was suggested that the formation of bishydrated-oxygen complex introduces partly filled defect states that cause energy level bending. However, we find that the shift in work function upon oxygen exposure of $\mathrm{P}(\mathrm{NDI} 2 \mathrm{OD}-\mathrm{T} 2)$ is independent of film thickness 12, 8 and $3 \mathrm{~nm}$ (see Fig.S2 and Table S1), so energy level bending due to the formation of an extended depletion region cannot be the cause of the observed shifts. The new states created by the $\mathrm{O}_{2} \bullet \bullet P(N D I 2 O D-T 2)$ complex formation also cannot explain the behavior, as although the HOMO is destabilized (in agreement with the decrease of the $\mathrm{E}_{\mathrm{ICT}+}$ ), the inter-gap state should yield an increased EICT-, which is not the case. We instead turn to the work function modification at doped conjugated polymer/electrode interface ${ }^{26}$ and conjugated electrolyte/electrode interface ${ }^{27}$, where one of the ions in the organic semiconductor film is more mobile than the counter one. At interface the Ion $^{+}$and Ion $^{-}$ 
can respectively interact with their induced image dipole of the substrate, one of the ion species are move closer to the substrate and thus form a "double dipole step” shifting the work function. ${ }^{27,} 28$ The effect does not depend on the substrate work function and only occurs at interface, hence is film thickness independent in line with our results. The overall energy level alignment of such systems then follows the ICT model modified by the double-dipole step (see supporting information). ${ }^{26-27}$ To further test if the work function shift of the ideal ICT curve in the P(NDI2OD-T2) film upon oxygen exposure can be explained by the similar mechanisms with a negatively charged $\mathrm{O}_{2}$ and positively charged $\mathrm{P}(\mathrm{NDI} 2 \mathrm{OD}-\mathrm{T} 2)$ in the $\mathrm{O}_{2} \bullet \bullet P(N D I 2 O D-T 2)$ complex, heating of the exposed film was carried out in ultrahigh vacuum. The original work function is recovered with no other change to the UPS spectra features (Fig. S3), indicating a weak interaction between oxygen molecule and $\mathrm{P}(\mathrm{NDI} 2 \mathrm{OD}-\mathrm{T} 2)$ as well as a reversible process as predicted by theory. This is further supported by XPS C1s, O1s and N1s core level spectra of P(NDI2OD-T2) film, as shown in Fig. 3, where no new features after 180 min oxygen exposure are observed. Note that the applied exposure time is sufficient for complete diffusion of oxygen molecules through the film. If the negatively charged oxygen molecules are more mobile than the positively charged P(NDI2OD-T2) in the $\mathrm{O}_{2} \bullet \bullet P(N D I 2 O D-T 2)$ complexes, we expect the formation of a "double dipole" step at the polymer-substrate interface that downshifts the work function regardless of initial substrate work function as is indeed the case, see Fig. 4 of the energy level alignment diagrams for $\mathrm{P}(\mathrm{NDI} 2 \mathrm{OD}-\mathrm{T} 2)$ interfaces before and after oxygen exposure, which is different with the case of the energy level shift originating from the gas molecule 
penetration into the film through the crystal-grain boundaries leading to the small imperfections of the packing structures and thus change of the gap states. ${ }^{29}$

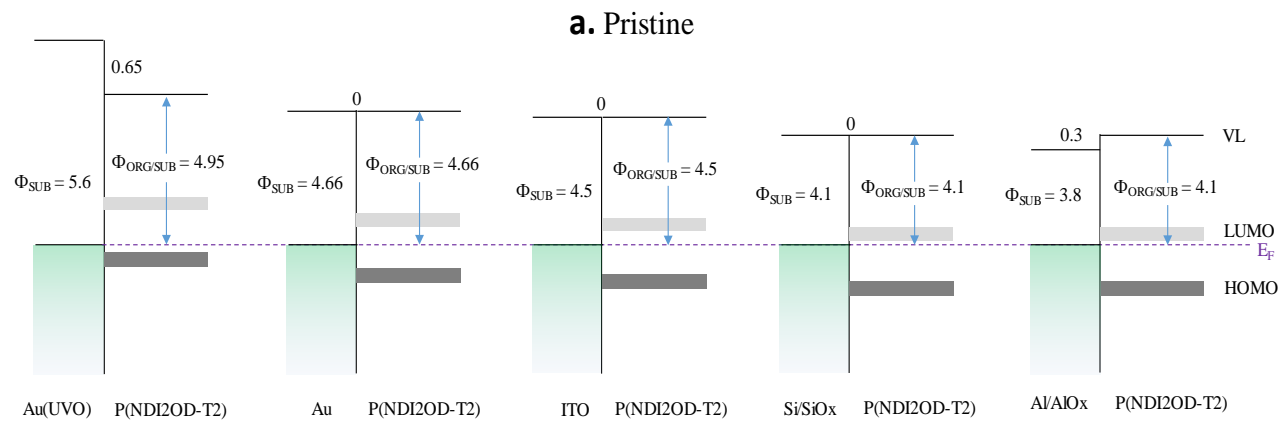

b. After

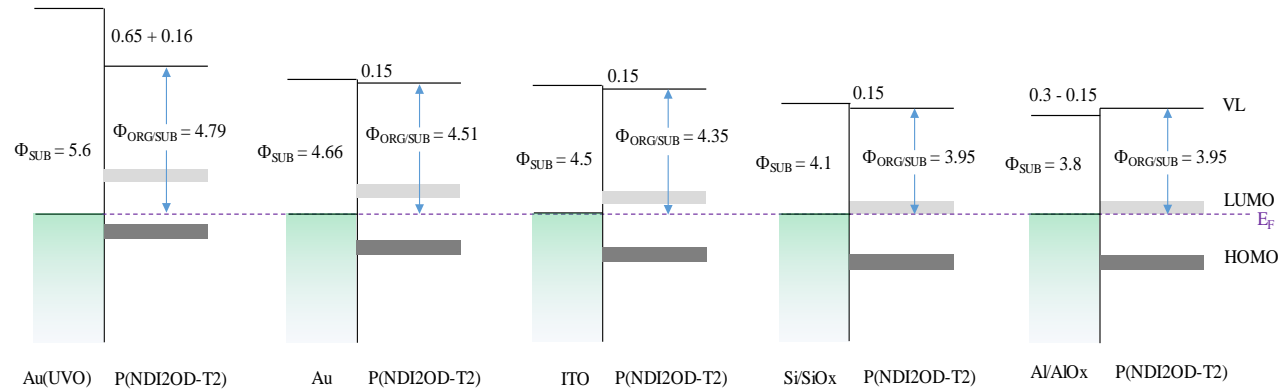

c. Double dipole step $\left(D_{1}+D_{2}\right)$

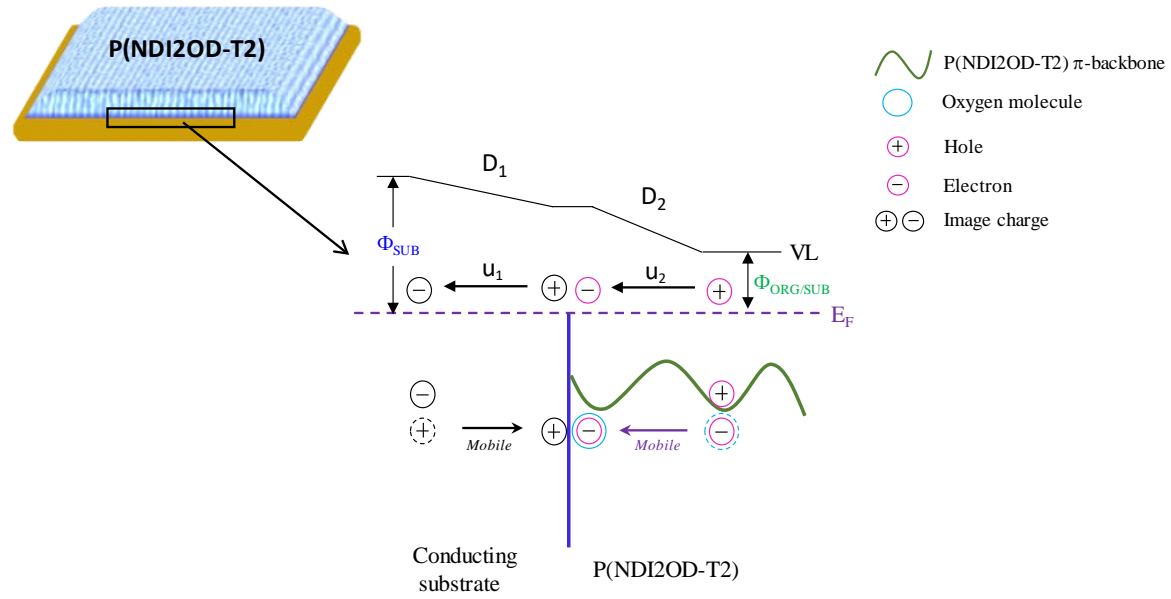

Figure 4. Energy level alignment diagrams showing the effect of oxygen absorption on P(NDI2OD-T2) interface energetics: (a) pristine and (b) after oxygen exposure. (c) Schematic illustration of work function downshift at interface upon oxygen exposure, which originates from the double dipole step $\left(D_{1}+D_{2}\right)$ formation at interface. In 
the oxygen-doped P(NDI2OD-T2) film, the diffused oxygen molecule is negatively charged (blue circle with dashed line), and the polymer $\pi$ backbone carries the holes (red circle with solid line). The electron and hole pairs in the P(NDI2OD-T2) film respectively developed the opposite image charge (black circle) in the conducting substrate across the interface. Related to the hole-carrying P(NDI2OD-T2), the negatively charged oxygen molecules are more mobile toward the interface. The purple arrow points to the oxygen molecule motion, resulting in electron redistribution at the interface (blue circle with solid line). This process produces two negative dipole moments $\mathrm{u}_{1}$ and $\mathrm{u}_{2}$ at the $\mathrm{P}(\mathrm{NDI} 2 \mathrm{OD}-\mathrm{T} 2) /$ conducting substrate interface, leading to diminish the work function $\left(\mathrm{D}_{1}+\mathrm{D}_{2}\right)$ of the film.

We expect the results present here also hold for other organic semiconductor featuring weak interaction with oxygen molecules (no covalent bonding) as long as the negatively charged oxygen in the complex is more mobile than the positive charge on the organic semiconductor. The analogous energetic modification phenomena in the p-type conjugated polymer poly[2,3-bis-(3-octyloxyphenyl) quinoxaline-5, 8-dilyl-altthiophene-2, 5-diyl] (TQ1) film upon oxygen exposure are also observed (see Fig. S4). Work function downshifts by $0.15 \mathrm{eV}$ under the pristine TQ1 ICT curve and no strong chemical interaction between polymer and oxygen molecule after saturated oxygen exposure is found (Fig. S4b-c, Fig. S5, Fig. S6 and Table S2). 
b
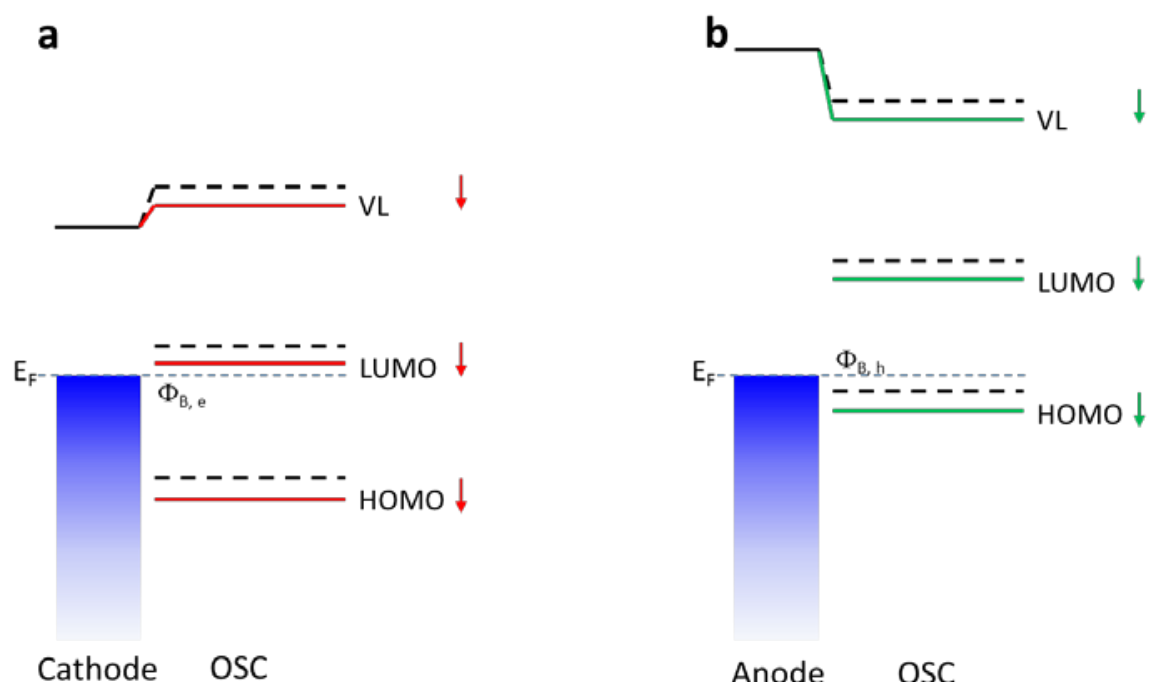

Cathode OSC

Figure 5. Charge injection barrier modification upon absorption of oxygen molecules at (a) cathode/organic semiconductor (OSC) contact and (b) anode/OSC contact. Dashed lines represent pristine OSC film vacuum and energy levels, colored solid lines represent OSC film vacuum and energy levels after oxygen exposure.

These energetics modifications upon oxygen exposure may have a significant effect on the performance of organic electronic devices. The formation of an additional double dipole step will decrease the overall interface dipole at a cathode (low work function contact, region (i) in Fig. S7) and increase the overall interface dipole at an anode (high work function contact, region (iii) in Fig. S7). Since the film IP is unchanged (and assuming the same is true for the electron affinity), this will move the LUMO level closer to Fermi level at cathode/organic semiconductor interface, decreasing the electron-injection barrier $\left(\Phi_{\mathrm{B}, \mathrm{e}}\right)$ here defined as the energy offset between $\mathrm{E}_{\mathrm{F}}$ and bulk LUMO level, ${ }^{30}$ see Fig. 5a. The energy level-matched contact is then expected to be more efficient for the electron injection, thereby increasing device performance. For anode/organic semiconductor interface in Fig. 5b, the addition of a double dipole step 
causes a larger hole-injection barrier $\left(\Phi_{\mathrm{B}, \mathrm{h}}\right)$, here defined as the energy offset between EF and bulk HOMO level, resulting in a loss in device performance.

\section{Conclusion}

In conclusion, we show the experimental evidences and theoretical explanations for the effects of in situ oxygen exposure on energetics in n-type polymer film, and discuss the origin of the charge injection barrier evolution at the electrode contact. We have found that all the work functions of the P(NDI2OD-T2) films regularly downshift by approximately $0.15 \mathrm{eV}$ below the original ICT curve upon oxygen exposure and their HOMO edges shift to higher binding energy by the same energy. The IP thus remains constant and there is no broadening of the spectral features. We attributed to these findings to the establishment of a so-called double dipole step via motion of charged molecules and will modify the charge injection barriers at electrode contact. The interaction between P(NDI2OD-T2) and oxygen molecules is weak (no direct bonds), and the negatively charged oxygen molecules are more mobile than the positively charged conjugated polymer. As a consequence, the double dipole step formed at interface downshifts the work function for all metal or semiconducting substrates. We further demonstrated that the process also holds for oxygen-exposed p-type polymer TQ1 films, indicating it may be a universal principle for organic semiconductor films that undergo weak interaction with oxygen molecules (no covalent bonding). The energetics modifications at anode and cathode contacts can affect the performance of organic electronic devices through modification of the charge injection barriers. We 
expect that the results have significance for the understanding and control of environmental factors in organic electronic devices.

\section{Experimental Section:}

Materials: The n-type polymer P(NDI2OD-T2) used in the study obtained from Polyera Company. The p-type polymer TQ1 was synthesized at the Chambers University of Technology, Sweden. Films from o-dichlorobenzene solution were spin-coated on different substrates in dark conduction, then directly and quickly transferred into the load lock of the ultrahigh vacuum (UHV) system. The substrates and the corresponding work functions are following: $\mathrm{AlOx} / \mathrm{Al}(\Phi=3.8 \mathrm{eV}), \mathrm{SiOx} / \mathrm{Si}(\Phi=4.1 \mathrm{eV}), \mathrm{ITO}(\Phi=$ 4.5 eV), AuOx/Au $(\Phi=4.66 \mathrm{eV})$, PEDOT: PSS $(\Phi=5.1 \mathrm{eV})$, and UVO treated Au $(\Phi$ $=5.6 \mathrm{eV})$. All substrates were cleaned by sonication in acetone and isopropanol before using.

Characterization of energetics evolution: Photoelectron spectroscopy measurements were performed in an UHV surface analysis system equipped with Scienta-200 hemispherical analyser. The system includes a load lock chamber with a base pressure of $1 \times 10^{-7} \mathrm{mbar}$, a preparation chamber with a base pressure of $8 \times 10^{-10} \mathrm{mbar}$, and an analysis chamber with a base pressure of $2 \times 10^{-10}$ mbar. In-situ exposure of P(NDI2ODT2) and TQ1 film to oxygen gas was carried in the preparation chamber, and the exposure pressure of $6 \times 10^{-6}$ mbar is controlled via a leak valve. After each cycle of oxygen exposure, the sample were transferred to the analysis chamber without breaking UHV for measurement. UPS was measured using HeI $21.22 \mathrm{eV}$ as excitation source with energy resolution of $0.05 \mathrm{eV}$ to investigate the work function and the frontier 
electronic structure features. The work function was derived from the secondary electron cut-off of the spectra, and the ionization potential is estimated from the frontier edge of the occupied density of states. XPS with monochromatized Al Ka $1486.6 \mathrm{eV}$ was used to detect possible chemical interaction after exposure. All spectra were calibrated with reference to the Fermi level and $\mathrm{Au} 4 \mathrm{f}_{7 / 2}$ peak position of the $\mathrm{Ar}^{+}$ion sputter-clean gold foil.

Acknowledgements: The work is sponsored by the National Science Foundation of China grant No.11604099, ECNU Team Building grant, the Fundamental Research Funds for the Central Universities, the Swedish Foundation for Strategic Research SE13-0060, the Swedish Research Council project grant 2016-05498, the Goran Gustafsson Foundation for Research in Nature Sciences and Medicine and the Swedish Government Strategic Research Area in Materials Science on Functional Materials at Linköping University (Faculty Grant SFO Mat LiU No 2009 00971). Q. Bao also thank the Open Project of Jiangsu Key Laboratory for Carbon-Based Functional Materials \& Devices.

\section{Supporting Information}

UPS of P(NDI2OD-T2) without oxygen exposure, work function of the increased film thickness, ICT curve and energetics evolution in TQ1film, XPS, a brief introduction of ICT model modified by double dipole step

\section{Author Information}


*Email: qybao@clpm.ecnu.edu.cn

The authors declare no competing financial interest.

\section{References:}

(1). Oh, J. Y.; Rondeau-Gagne, S.; Chiu, Y. C.; Chortos, A.; Lissel, F.; Wang, G. J. N.; Schroeder, B. C.; Kurosawa, T.; Lopez, J.; Katsumata, T.; Xu, J.; Zhu, C. X.; Gu, X. D.; Bae, W. G.; Kim, Y.; Jin, L. H.; Chung, J. W.; Tok, J. B. H.; Bao, Z. N., Intrinsically Stretchable and Healable Semiconducting Polymer for Organic Transistors. Nature 2016, 539, 411-415.

(2). Sirringhaus, H.; Kawase, T.; Friend, R. H.; Shimoda, T.; Inbasekaran, M.; Wu, W.; Woo, E. P., HighResolution Inkjet Printing of All-polymer Transistor Circuits. Science 2000, 290, 2123-2126.

(3). Hofle, S.; Schienle, A.; Bruns, M.; Lemmer, U.; Colsmann, A., Enhanced Electron Injection into Inverted Polymer Light-Emitting Diodes by Combined Solution-Processed Zinc Oxide/Polyethylenimine Inter layers. Adv. Mater. 2014, 26, 2750-2754.

(4). Bolink, H. J.; Coronado, E.; Orozco, J.; Sessolo, M., Efficient Polymer Light-Emitting Diode Using Air-Stable Metal Oxides as Electrodes. Adv. Mater. 2009, 21, 79-82.

(5). Dennler, G.; Scharber, M. C.; Brabec, C. J., Polymer-Fullerene Bulk-Heterojunction Solar Cells. Adv. Mater. 2009, 21, 1323-1338.

(6). Lin, Y.; Zhao, F.; Wu, Y.; Chen, K.; Xia, Y.; Li, G.; Prasad, S. K.; Zhu, J.; Huo, L.; Bin, H.; Zhang, Z. G.; Guo, X.; Zhang, M.; Sun, Y.; Gao, F.; Wei, Z.; Ma, W.; Wang, C.; Hodgkiss, J.; Bo, Z.; Inganas, O.; Li, Y.; Zhan, X., Mapping Polymer Donors toward High-Efficiency Fullerene Free Organic Solar Cells. Adv. Mater. 2017, 29, 1604155.

(7). Braun, S.; Salaneck, W. R.; Fahlman, M., Energy-Level Alignment at Organic/Metal and Organic/Organic Interfaces. Adv. Mater. 2009, 21, 1450-1472.

(8). Bao, Q. Y.; Yang, J. P.; Xiao, Y.; Deng, Y. H.; Lee, S. T.; Li, Y. Q.; Tang, J. X., Correlation Between the Electronic Structures Of Transition Metal Oxide-based Intermediate Connectors and the Device Performance of Tandem Organic Light-emitting Devices. J. Mater. Chem. 2011, 21, 17476 17482.

(9). Meyer, J.; Hamwi, S.; Kroger, M.; Kowalsky, W.; Riedl, T.; Kahn, A., Transition Metal Oxides for Organic Electronics: Energetics, Device Physics and Applications. Adv. Mater. 2012, 24, 5408-5427. (10). Wang, Y.; Di Motta, S.; Negri, F.; Friedlein, R., Effect of Oxygen on the Electronic Structure of Highly Crystalline Picene Films. J. Am. Chem. Soc. 2011, 133, 10054-10057.

(11). Zhong, J. Q.; Mao, H. Y.; Wang, R.; Lin, J. D.; Zhao, Y. B.; Zhang, J. L.; Ma, D. G.; Chen, W., Ionization Potential Dependent Air Exposure Effect on the MoO3/Organic Interface Energy Level Alignment. Org. Electron. 2012, 13, 2793-2800.

(12). Aygul, U.; Hintz, H.; Egelhaaf, H. J.; Distler, A.; Abb, S.; Peisert, H.; Chasse, T., Energy Level Alignment of a P3HT/Fullerene Blend during the Initial Steps of Degradation. J. Phys. Chem. C 2013, 117, 4992-4998.

(13). Nicolai, H. T.; Kuik, M.; Wetzelaer, G. A. H.; de Boer, B.; Campbell, C.; Risko, C.; Bredas, J. L.; Blom, P. W. M., Unification of Trap-limited Electron Transport in Semiconducting Polymers. Nature Mater. 2012, 11, 882-887. 
(14). Hintz, H.; Peisert, H.; Egelhaaf, H. J.; Chasse, T., Reversible and Irreversible Light-Induced pDoping of P3HT by Oxygen Studied by Photoelectron Spectroscopy (XPS/UPS). J. Phys. Chem. C 2011, 115, 13373-13376.

(15). Bhattacharya, J.; Mayer, R. W.; Samiee, M.; Dalal, V. L., Photo-induced Changes in Fundamental Properties of Organic Solar Cells. Appl. Phys. Lett. 2012, 100, 193501.

(16). Sirringhaus, H., Reliability of Organic Field-Effect Transistors. Adv. Mater. 2009, 21, 3859 3873.

(17). Yan, H.; Chen, Z. H.; Zheng, Y.; Newman, C.; Quinn, J. R.; Dotz, F.; Kastler, M.; Facchetti, A., A High-mobility Electron-transporting Polymer for Printed Transistors. Nature 2009, 457, 679-686.

(18). Xia, Y.; Musumeci, C.; Bergqvist, J.; Ma, W.; Gao, F.; Tang, Z.; Bai, S.; Jin, Y.; Zhu, C.; Kroon, R.; Wang, C.; Andersson, M. R.; Hou, L.; Inganäs, O.; Wang, E., Inverted All-polymer Solar Cells Based on a Quinoxaline-thiophene/naphthalene-diimide Polymer Blend Improved by Annealing. J. Mater. Chem. A 2016, 4, 3835-3843.

(19). Di Pietro, R.; Fazzi, D.; Kehoe, T. B.; Sirringhaus, H., Spectroscopic Investigation of Oxygenand Water-Induced Electron Trapping and Charge Transport Instabilities in n-type Polymer Semiconductors. J. Am. Chem. Soc. 2012, 134, 14877-14889.

(20). Fahlman, M.; Crispin, A.; Crispin, X.; Henze, S. K. M.; de Jong, M. P.; Osikowicz, W.; Tengstedt, C.; Salaneck, W. R., Electronic Structure of Hybrid Interfaces for Polymer-based Electronics. J. PhysCondens Mat. 2007, 19, 183202.

(21). Bao, Q. Y.; Sandberg, O.; Dagnelund, D.; Sanden, S.; Braun, S.; Aarnio, H.; Liu, X. J.; Chen, W. M. M.; Osterbacka, R.; Fahlman, M., Trap-Assisted Recombination via Integer Charge Transfer States in Organic Bulk Heterojunction Photovoltaics. Adv. Funct. Mater. 2014, 24, 6309-6316.

(22). Greiner, M. T.; Helander, M. G.; Tang, W. M.; Wang, Z. B.; Qiu, J.; Lu, Z. H., Universal EnergyLevel Alignment of Molecules on Metal Oxides. Nature Mater. 2012, 11, 76-81.

(23). Ley, L.; Smets, Y.; Pakes, C. I.; Ristein, J., Calculating the Universal Energy-Level Alignment of Organic Molecules on Metal Oxides. Adv. Funct. Mater. 2013, 23, 794-805.

(24). Fahlman, M.; Sehati, P.; Osikowicz, W.; Braun, S.; de Jong, M. P.; Brocks, G., Photoelectron Spectroscopy and Modeling of Interface Properties Related to Organic Photovoltaic Cells. J. Electron Spectrosc. Rela. Phenom. 2013, 190, 33-41.

(25). Bao, Q. Y.; Liu, X. J.; Braun, S.; Fahlman, M., Oxygen-and Water-Based Degradation in [6,6]Phenyl-C-61-Butyric Acid Methyl Ester ( PCBM) Films. Adv. Energy Mater. 2014, 4, 1301272.

(26). Bao, Q. Y.; Liu, X. J.; Braun, S.; Gao, F.; Fahlman, M., Energetics at Doped Conjugated Polymer/Electrode Interfaces. Adv. Mater. Interfaces 2015, 2, 1400403.

(27). Bao, Q.; Liu, X.; Wang, E.; Fang, J.; Gao, F.; Braun, S.; Fahlman, M., Regular Energetics at Conjugated Electrolyte/Electrode Modifier for Organic Electronics and their Implications on Design Rules. Adv. Mater. Interfaces 2015, 2, 1500204.

(28). Van Reenen, S.; Kouijzer, S.; Janssen, R. A. J.; Wienk, M. M.; Kemerink, M., Origin of Work Function Modification by lonic and Amine-Based Interface Layers. Adv. Mater. Interfaces 2014, 1, 1400189.

(29). Bussolotti, F.; Kera, S.; Kudo, K.; Kahn, A.; Ueno, N., Gap States in Pentacene Thin Film Induced by Inert Gas Exposure. Phys Rev Lett 2013, 110, 267602.

(30). Atxabal, A.; Braun, S.; Arnold, T.; Sun, X.; Parui, S.; Liu, X.; Gozalvez, C.; Llopis, R.; Mateo-Alonso, A.; Casanova, F.; Ortmann, F.; Fahlman, M.; Hueso, L. E., Energy Level Alignment at Metal/SolutionProcessed Organic Semiconductor Interfaces. Adv. Mater. 2017, 29, 1606901. 


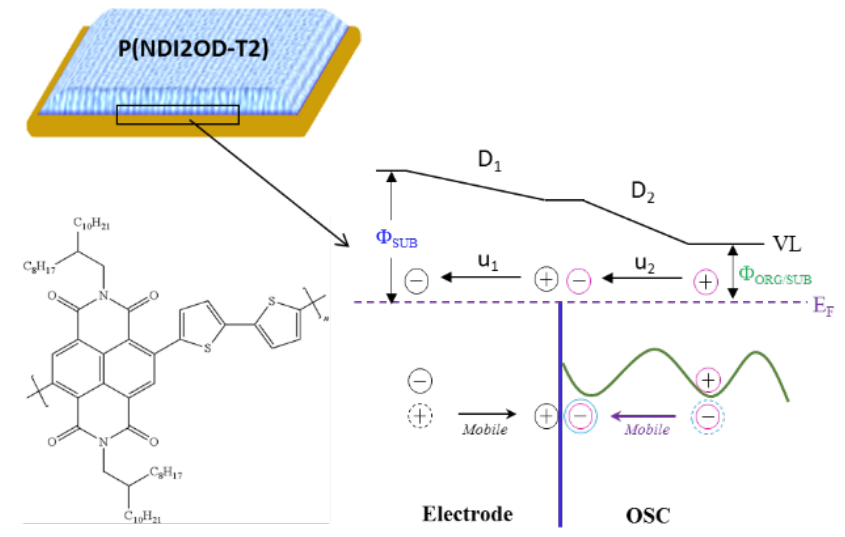




\section{Supporting Information}

\section{The Effect of Oxygen Uptake on Charge Injection Barriers in Conjugated Polymer Films}

Qinye Bao ${ }^{1,2,3^{*}}$, Xianjie $\mathrm{Liu}^{2}$, Slawomir Braun ${ }^{2}$, Jianming Yang ${ }^{1}$, Yanqing $\mathrm{Li}^{4}$, Jianxin Tang $^{4}$, Chungang Duan ${ }^{1}$, Mats Fahlman ${ }^{2}$

1 Key Laboratory of Polar Materials and Devices, Ministry of Education, East China Normal University, 200241, Shanghai, P.R. China

*E-mail: qybao@clpm.ecnu.edu.cn

2 Division of Surface Physics and Chemistry, IFM, Linköping University SE-58183 Linköping, Sweden

3 Jiangsu Key Laboratory for Carbon-Based Functional Materials \& Devices, Soochow University, Suzhou 215123, P. R. China

4 Institute of Functional Nano \& Soft Materials (FUNSOM), Soochow University, Suzhou 215123, P. R. China 


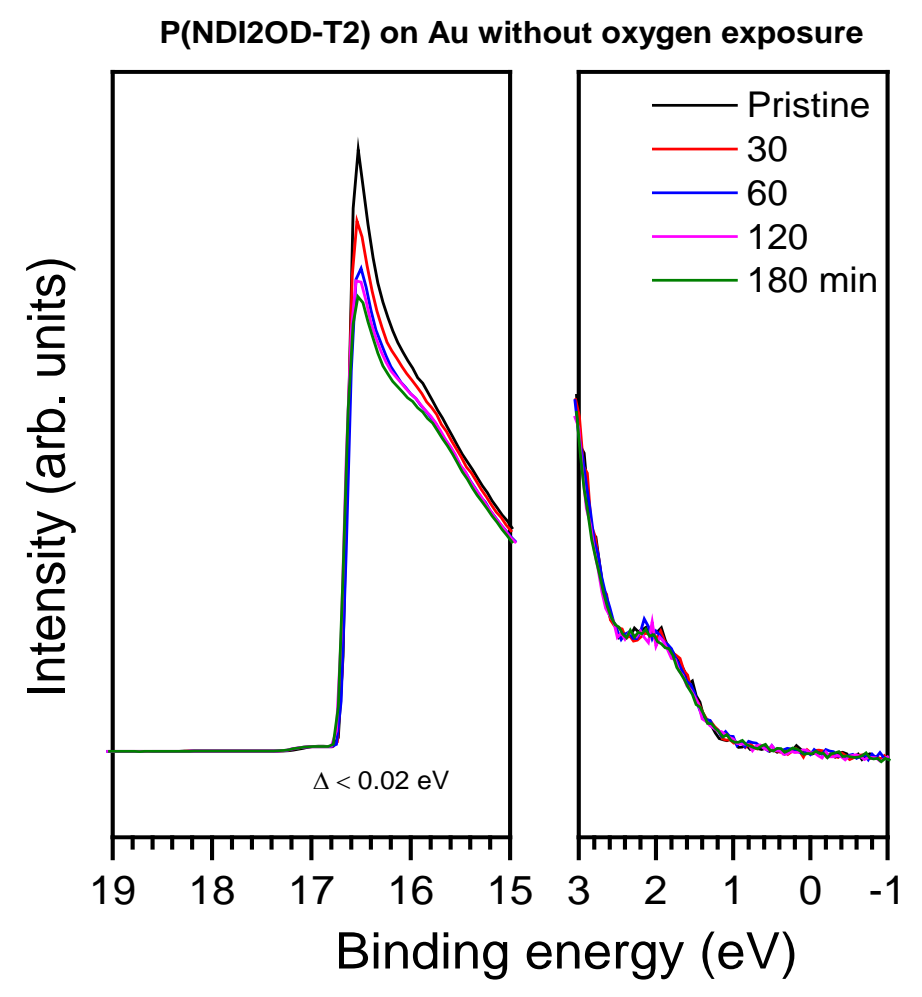

Figure S1. UPS spectra of the secondary electron region (work function, right) and the frontier electronic structure region (HOMO, left) of P(NDI2OD-T2) film as a function of time 30, 60, 120 and 180 min under the condition of no oxygen gas exposure. There is no change in the spectra features, implying that the UV lamp source during UPS measurement has no effect on the energetics of the P(NDI2OD-T2) film and the energetics evolution of the films in the main text indeed are attributed to the oxygen. 


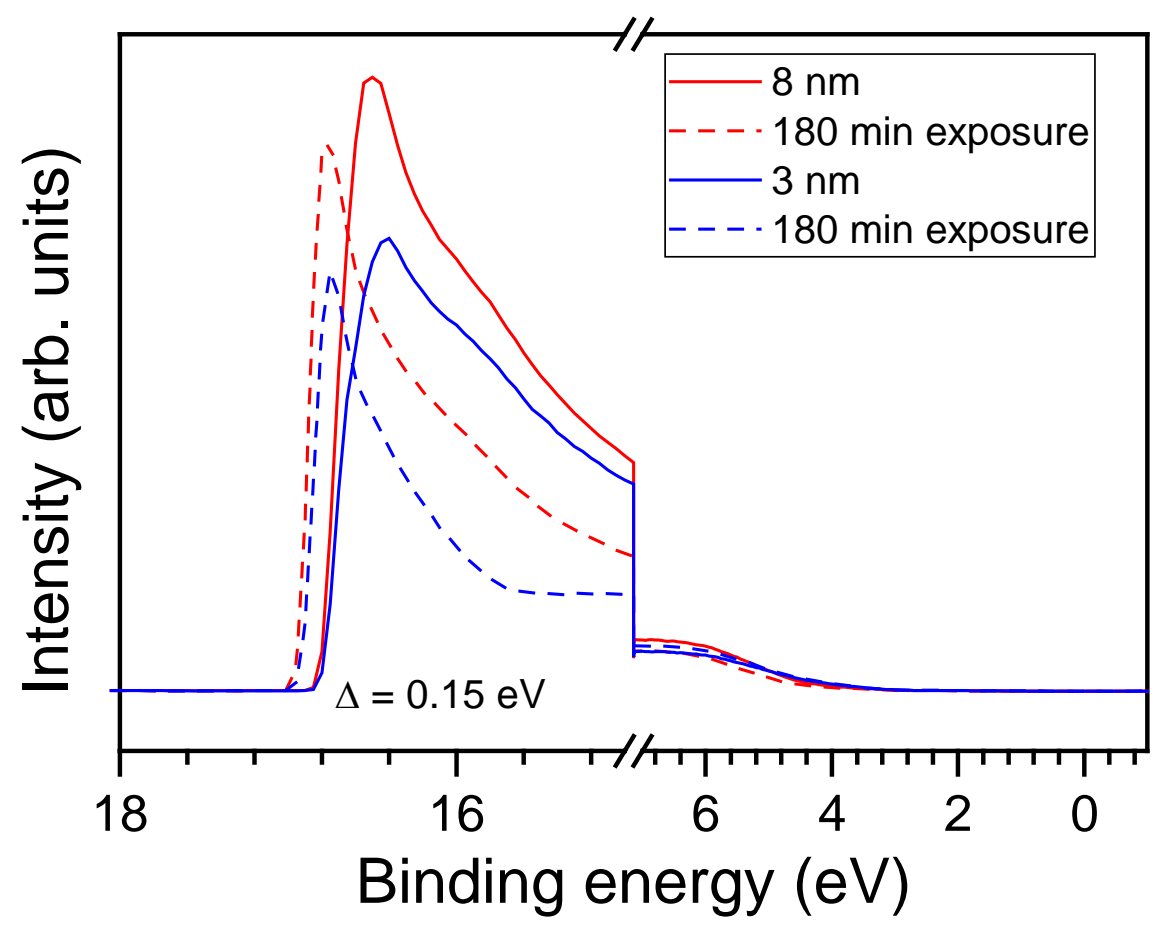

Figure S2. UPS spectra evolution of P(NDI2OD-T2) film coated on ITO with different thickness before (solid lines) and after (dashed lines) 180 min oxygen exposure. 
Table S1. Pristine work function (WF), WF after 180 min exposure, and WF shift $(\triangle)$ of P(NDI2OD-T2) films with different thickness 12, 8 and $3 \mathrm{~nm}$.

\begin{tabular}{|c|c|c|c|c|}
\hline $\begin{array}{c}\text { P(NDI2OD-T2) film } \\
\text { thickness (nm) }\end{array}$ & Substrate & $\begin{array}{c}\text { Pristine WF } \\
\mathbf{( e V )}\end{array}$ & $\begin{array}{c}\text { WF after 180 } \\
\text { min exposure } \\
\mathbf{( e V )}\end{array}$ & $\Delta \mathbf{( e V )}$ \\
\hline 12 [main text] & ITO & 4.5 & 4.35 & 0.15 \\
\hline 8 & ITO & 4.43 & 4.28 & 0.15 \\
\hline 3 & ITO & 4.45 & 4.3 & 0.15 \\
\hline
\end{tabular}




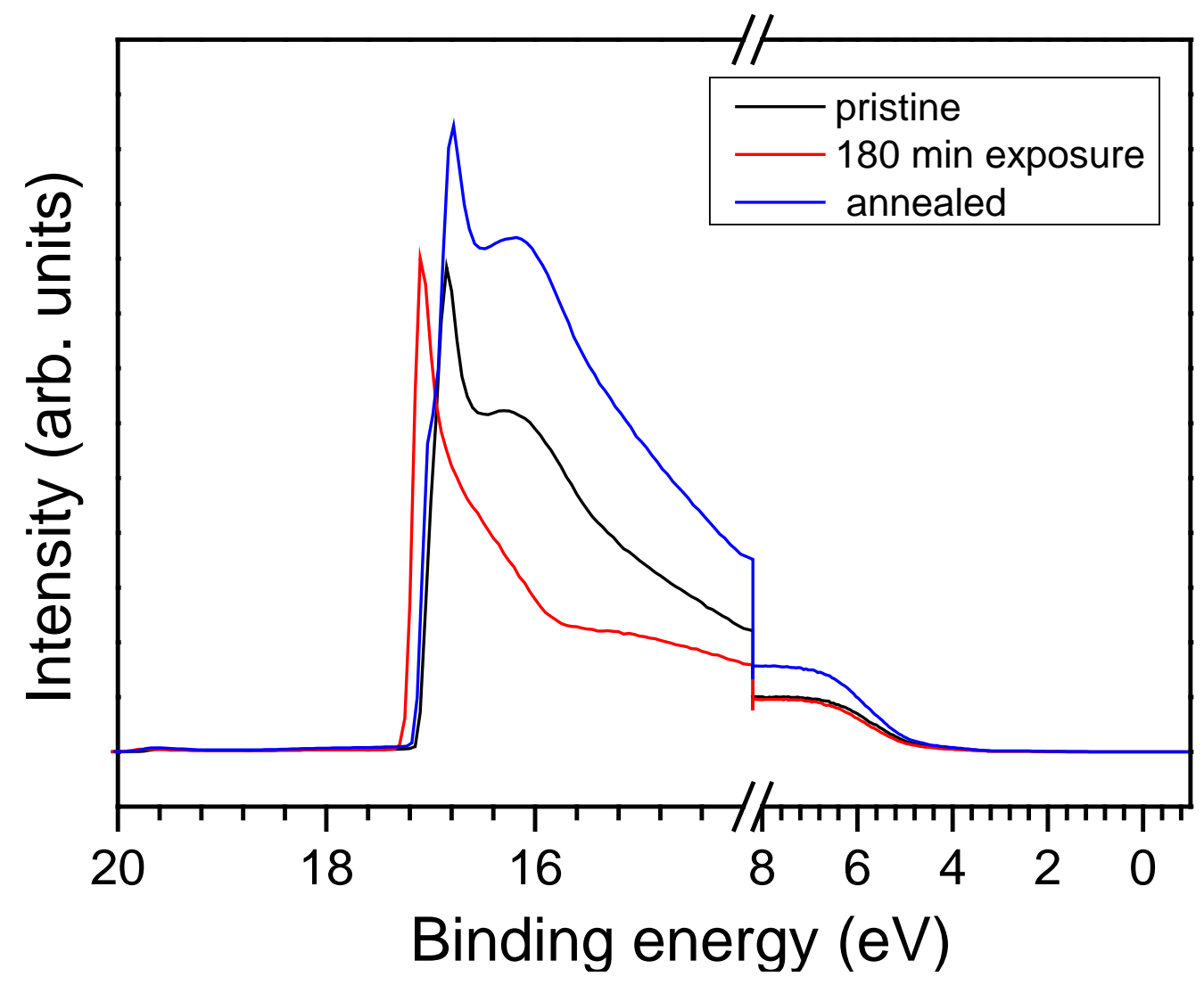

Figure S3. UPS spectra evolution of the pristine, oxygen-exposed and annealed $\mathrm{P}(\mathrm{NDI} 2 \mathrm{OD}-\mathrm{T} 2)$ film coated on AlOx/Al. The corresponding work functions are 4.1, 3.96 and $4.08 \mathrm{eV}$, respectively. 
Poly[2,3-bis-(3-octyloxyphenyl) quinoxaline-5, 8-dilyl-alt-thiophene-2, 5-diyl] (TQ1) is commonly used in organic photovoltaics as p-type donor polymer featuring high power conversion efficiency [1].

a

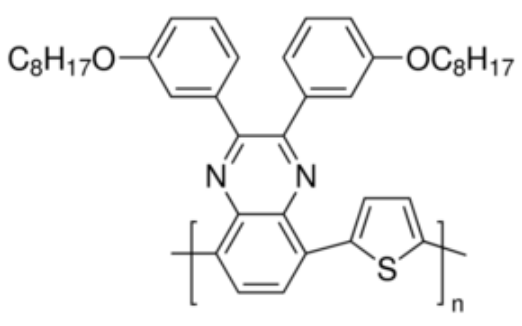

TQ1

\section{C}

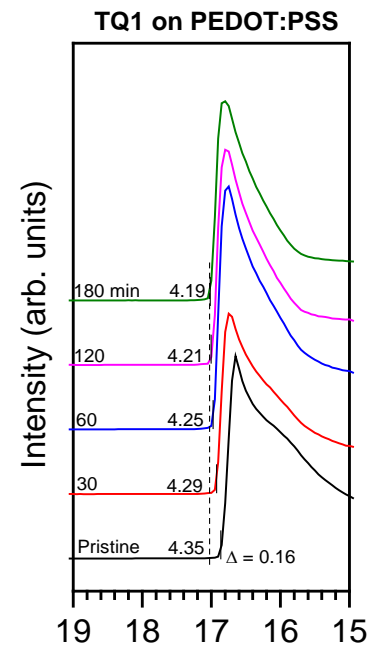

Binding energy (eV) b

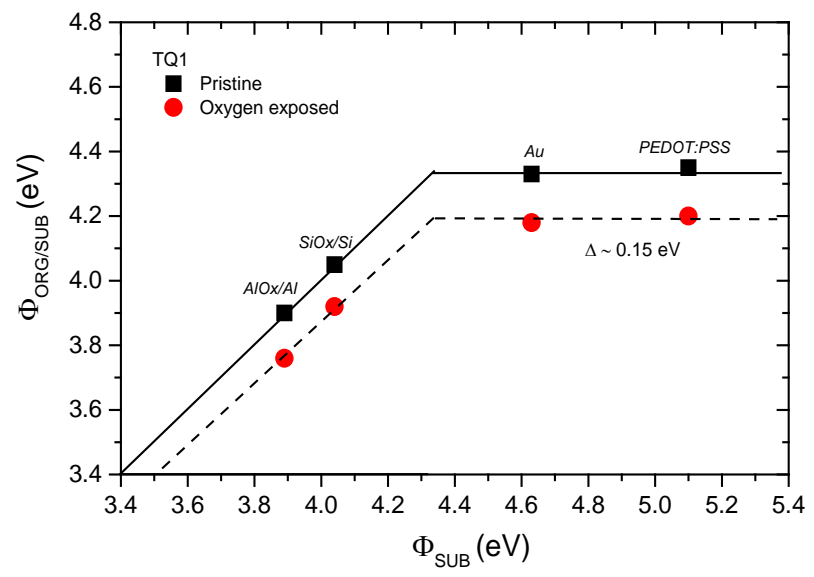

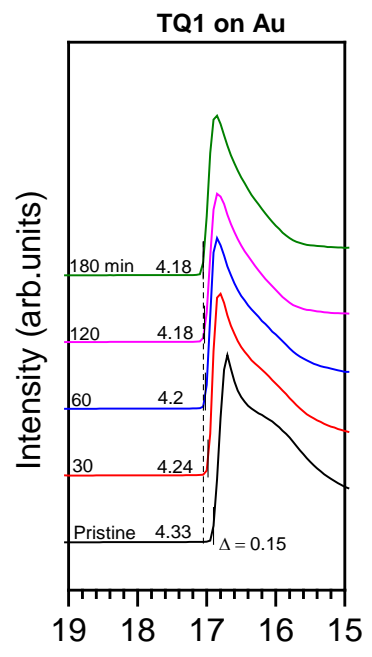

Binding energy $(\mathrm{eV})$

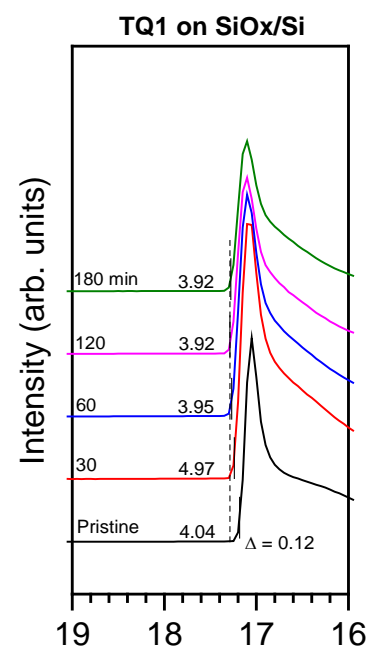

Binding energy $(\mathrm{eV})$

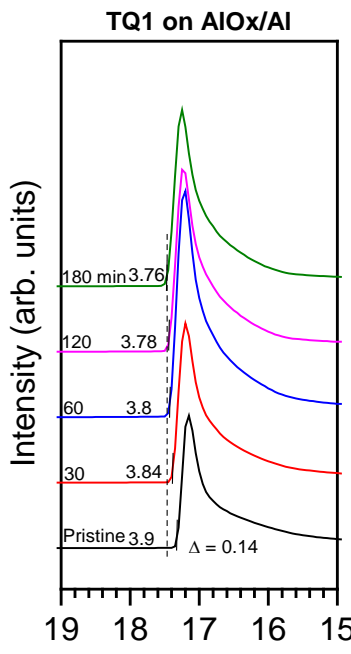

Binding energy (eV)

Figure S4. Energetics evolution phenomena in TQ1 film upon oxygen exposure. (a) chemical structure of TQ1, (b) The resulting ICT plots after oxygen exposure are downshifted by $\sim 0.15 \mathrm{eV}$ compared to the ICT plots of pristine TQ1, (c) Work function evolutions of UPS spectra of TQ1 film on various conducting substrates including PEDOT:PSS, Au, SiOx/Si and AlOx/Al, as a function of in situ oxygen gas exposure time 30, 60, 120 and 180 min. 

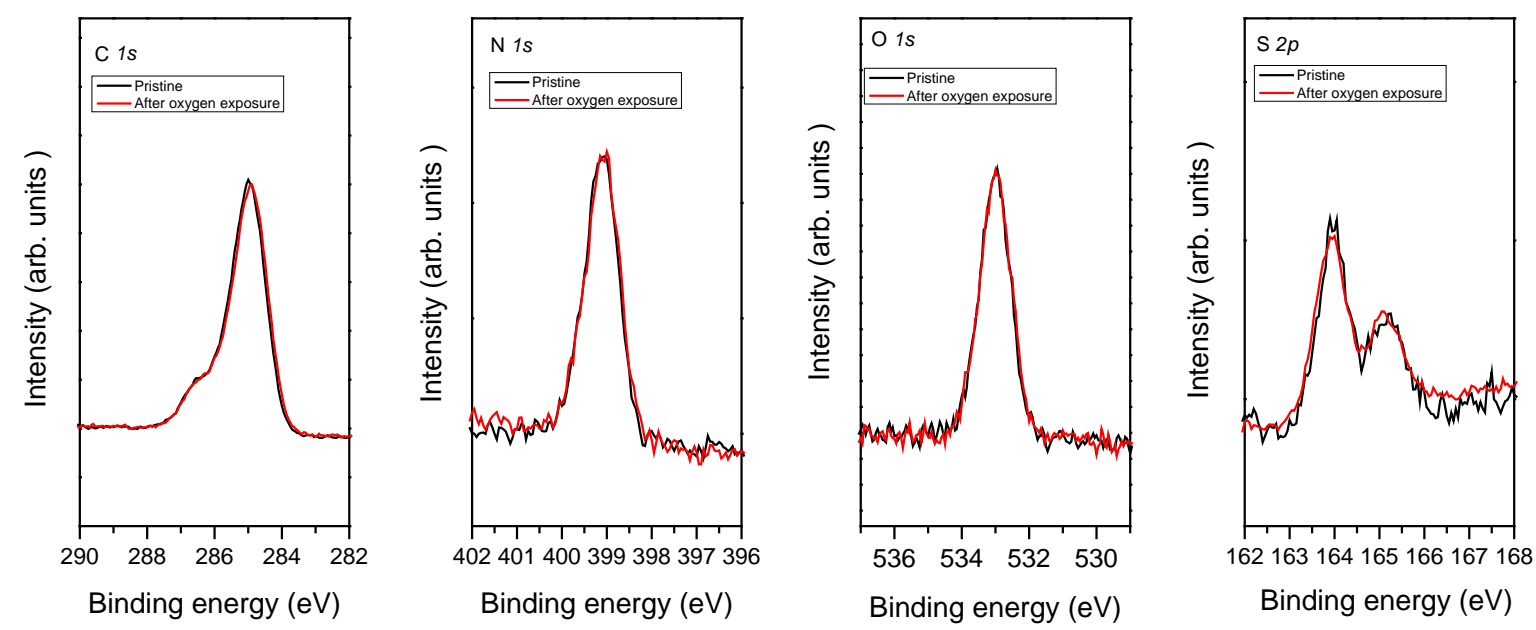

Figure S5. Comparison of XPS core level spectra C1s, O1s, N1s and S2p of TQ1 film before and after 180 min oxygen gas exposure. 


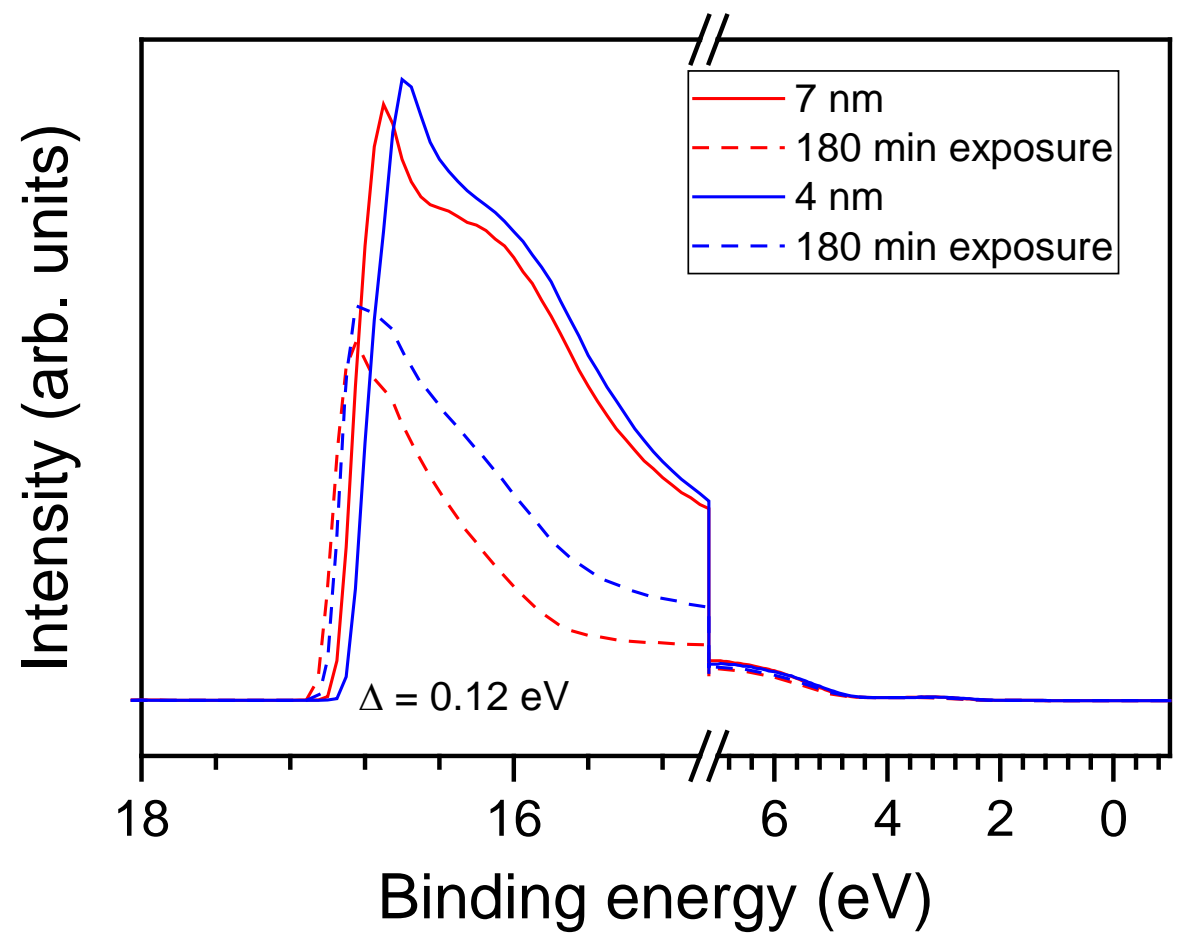

Figure S6. UPS spectra evolution of TQ1 films coated on ITO with different thickness before and after 180 min oxygen exposure. 
Table S2. Pristine work function (WF), WF after 180 min exposure, and WF shift $(\triangle)$ of TQ1 film with different thickness15, 7 and $4 \mathrm{~nm}$.

\begin{tabular}{|c|c|c|c|c|}
\hline $\begin{array}{c}\text { TQ1 film } \\
\text { thickness (nm) }\end{array}$ & Substrate & $\begin{array}{c}\text { Prinste WF } \\
\mathbf{( e V )}\end{array}$ & $\begin{array}{c}\text { WF after 180 } \\
\mathbf{m i n} \text { exposure } \\
\mathbf{( e V )}\end{array}$ & $\boldsymbol{\Delta} \mathbf{( e V )}$ \\
\hline 15 [main text] & - & - & - & $0.12-0.16$ \\
\hline 7 & ITO & 4.28 & 4.16 & 0.12 \\
\hline 4 & ITO & 4.33 & 4.20 & 0.13 \\
\hline
\end{tabular}




\section{ICT model modified by double dipole step:}

ICT model: The Integer Charge Transfer (ICT) model [2-8] can successfully describe and predict the equilibrium energy level alignment behavior at weakly interacting interfaces. It features an abrupt transition between a Schottky-Mott regime and Fermilevel pinning regimes upon variation of work function of underlying substrate to create interfaces spanning low to high work function, see Fig. S6. The Schottky-Mott regime (ii) is defined by vacuum level alignment while Fermi level pinning regimes (i and iii) feature the formation of a potential step that scales with difference between the equilibrium oxidation or reduction energies of the conjugated polymer at the interface and the work function of the substrate. The origin of the potential step is explained by spontaneous charge transfer across the interface via tunneling (integer charge transfer) when the substrate work function is greater (lower) than the energy required to oxidize (gained from reducing) a polymer segment at the interfaces. The ICT model states originate form oxidization/reduction energies at interface that strongly depend on the inter and intramolecular order [3,4], generally following the Gaussian distribution. [6,8] Sometimes such states are also referred to as tail states or gap states. The most easily oxidized/reduced of $\pi$-delocalized backbone adjacent to the interface hence will be "used up" creating integer charge transfer states until enough charge has been transferred across the interface to create a potential step that equilibrates the Fermi level. The energy where the Fermi level is subsequently pinned is referred to as EICT+,depending on if it is positive or negative polarons that are being created. The resulting three distinct energy level alignment regimes as per the ICT model are described by:

(i) $\Phi_{\text {SUB }}<E_{\text {ICT- }}$ - Fermi level pinning to a negative integer charge transfer state via integer electron spontaneously flowing from substrate to polymer, resulting in a substrate-independent work function, $\Phi_{\mathrm{ORG} / \mathrm{SUB}}=\mathrm{E}_{\mathrm{ICT}}$, , slope $=0$;

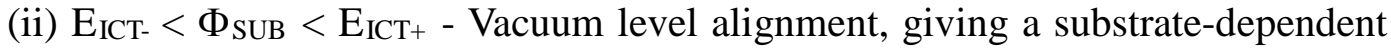
work function without charge transfer, $\Phi_{\mathrm{ORG} / \mathrm{SUB}}=\Phi_{\mathrm{SUB}}$, slope $=1$;

(iii) $\Phi_{\text {SUB }}>E_{\text {ICT+ }}$ - Fermi level pinning to a positive integer charge transfer state via integer spontaneously flowing from polymer to substrate, resulting again in a 
substrate-independent work function, $\Phi_{\mathrm{ORG} / \mathrm{SUB}}=\mathrm{E}_{\mathrm{ICT}+}$, slope $=0$.

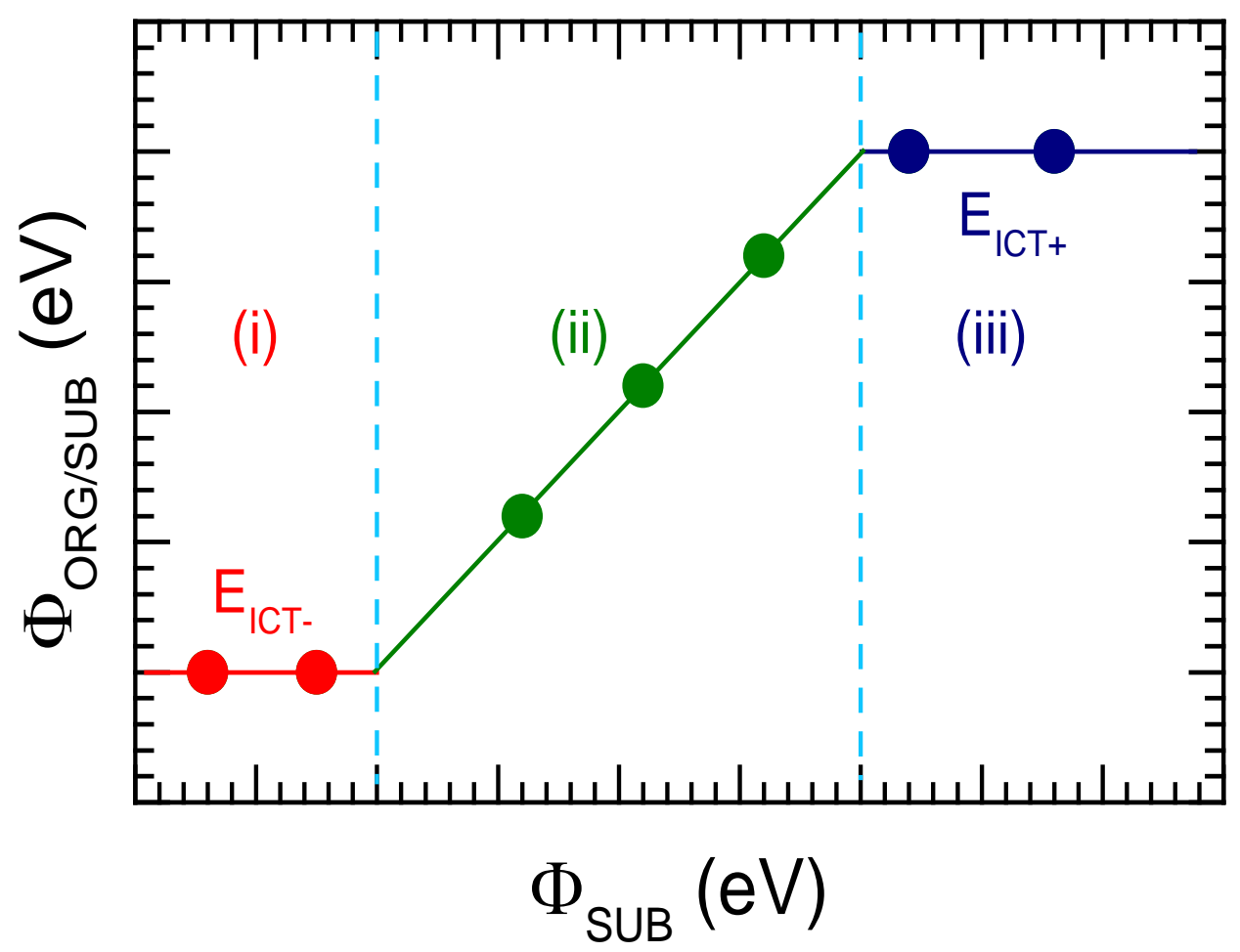

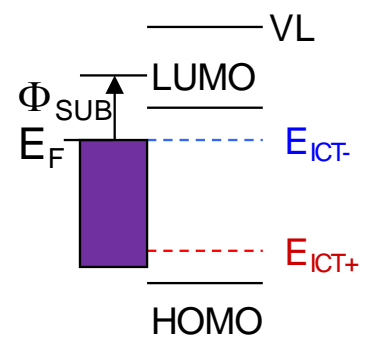

(i)

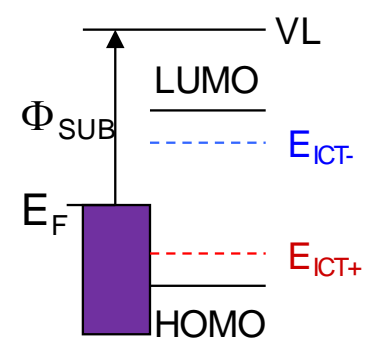

(ii)

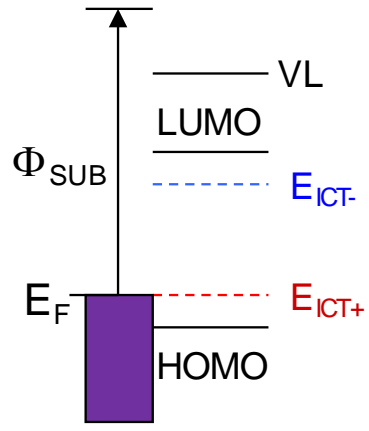

(iii)

Figure S7. Energy level alignment regimes (i, ii and iii) as per the ICT model.

Double dipole step: The concept of a double dipole step was proposed by Reenen et al

[9] to explain the work function modification in electrolyte-coated electrodes where one of the charged species (ions) can achieve a more intimate contact with the electrode surface by e.g. being more mobile than the counter charge. When the ions are located close to the interface, the $\operatorname{Ion}^{+}$and $\operatorname{Ion}^{-}$can interact with their respective induced image 
charges on the substrate, and since one of the ion species are more mobile, e.g. Ion $^{+}$, those ions will move closer to the substrate and as a consequence form a "double dipole step” up-shifting the work function. If the Ion- is more mobile, a double dipole step down-shifting the work function instead is formed. This effect only occurs at the interface and hence is film thickness independent, see the Fig. 4c in main text.

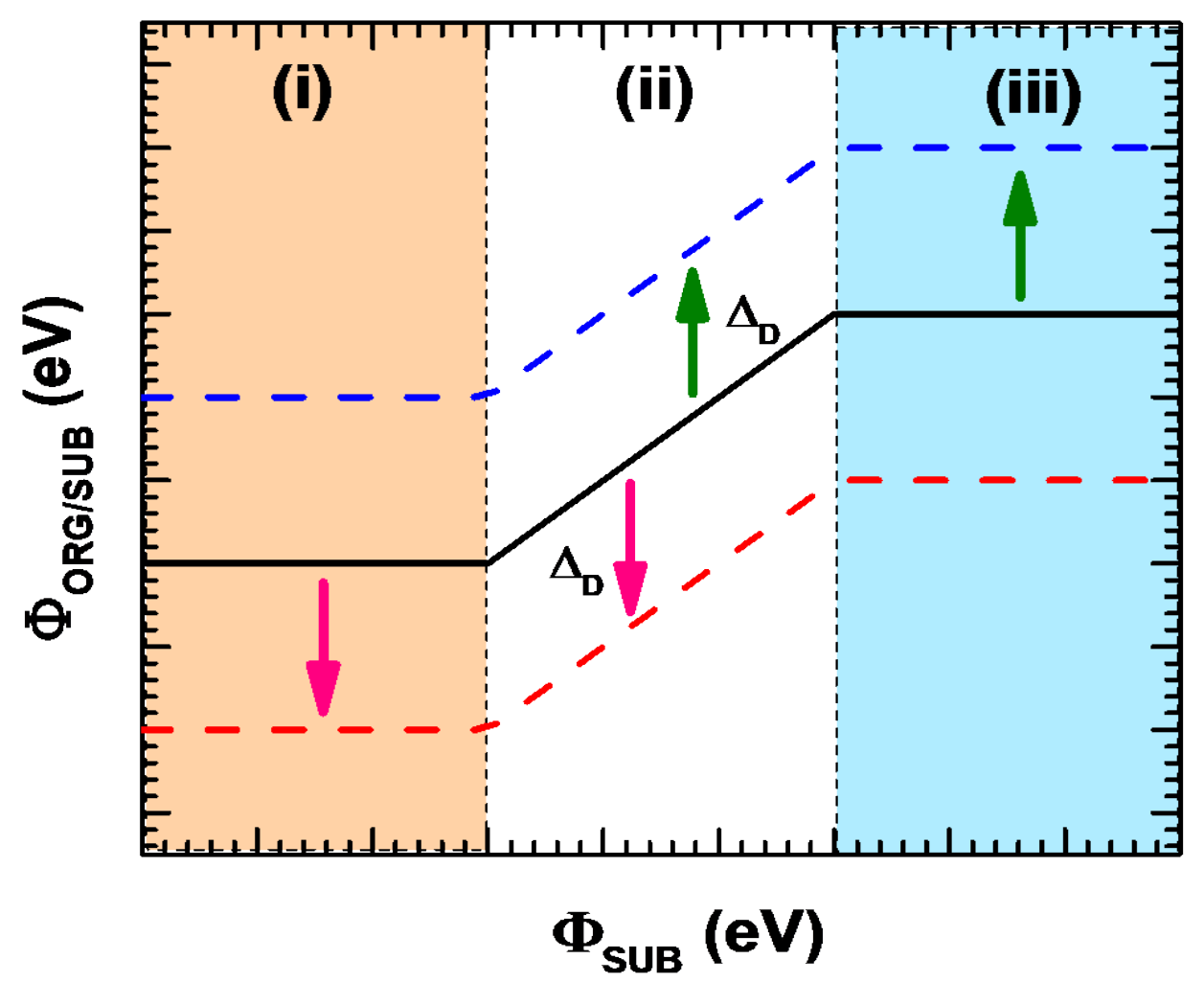

Figure S8. Shift of the ideal ICT curve (Solid black line) due to the double dipole step $\triangle_{\mathrm{D}}$.[10] The dash line means the resulted ICT curve by modification of the double dipole step.

ICT model modified by double dipole step: Recently, we demonstrated that the ICT model is modified by the double dipole step with the constant shift of the regular ICT curve featuring p-type doped organic semiconductors and conjugated electrolyte materials (see Fig. S7) [10, 11]. In the former case of F4TCNQ: rr-P3HT coulombicbound charge-transfer complexes form with a hole on the rr-P3HT and an electron on F4TCNQ. Since the holes on the rr-P3HT chains are more mobile/delocalized than the 
electrons on the much smaller F4TCNQ molecules, the double dipole effect occurs causing a work function shift for the (i), (ii) and (iii) regimes in the ICT curve. Also for conjugated (polymer) electrolyte materials, the double dipole step shifts the whole ICT curve yielding an increase or decrease of the work function of the electrode, depending on if the mobile charge is cationic or anionic. The energy level alignment at such interfaces is controlled by the equilibration of the Femi level due to oxidation/reduction of $\pi$-delocalized backbone as described by ICT model in combination additional double duple step at interface, up- or downshifting the "ideal” ICT curve.

\section{References:}

[1] Wang, E. G.; Hou, L. T.; Wang, Z. Q.; Hellstom, S.; Zhang, F. L.; Inganas, O.; Andersson, M. R., An Easily Synthesized Blue Polymer for High-Performance Polymer Solar Cells Adv. Mater. 2010, 22, 5240-5244.

[2] Braun, S.; Salaneck, W. R.; Fahlman, M., Energy-Level Alignment at Organic/Metal and Organic/Organic Interfaces. Adv. Mater. 2009, 21, 1450-1472.

[3] Fahlman, M.; Crispin, A.; Crispin, X.; Henze, S. K. M.; de Jong, M. P.; Osikowicz, W.; Tengstedt, C.; Salaneck, W. R., Electronic Structure of Hybrid Interfaces for Polymer-based Electronics. J. Phys-Condens Mat. 2007, 19, 183202.

[4] Sehati, P.; Braun, S.; Lindell, L.; Liu, X. J.; Andersson, L. M.; Fahlman, M, Energy Level Alignment at Metal-Organic and Organic-Organic Interfaces in BulkHeterojunction Solar Cells. IEEE J. Sel. Top Quant. 2010, 16, 1718-1724

[5] Greiner, M. T.; Helander, M. G.; Tang, W. M.; Wang, Z. B.; Qiu, J.; Lu, Z. H.; Universal Energy-level Alignment of Molecules on Metal Oxides, Nature Mater. 2012, $11,76-81$.

[6] Bao, Q. Y.; Sandberg, O.; Dagnelund, D.; Sanden, S.; Braun, S.; Aarnio, H.; Liu, X. J.; Chen, W. M. M.; Osterbacka, R.; Fahlman, M., Trap-Assisted Recombination via Integer Charge Transfer States in Organic Bulk Heterojunction Photovoltaics. Adv. Funct. Mater. 2014, 24, 6309-6316

[7] Tengstedt, C.; Osikowicz, W.; Salaneck, W. R.; Parker, I. D.; Hsu, C. H.; Fahlman, 
M. Fermi-level Pinning at Conjugated Polymer Interfaces, Appl. Phys. Lett. 2006, 88, 053502

[8] Oehzelt, M.; Koch, N.; Heimel, G., Organic Semiconductor Density of States Controls the Energy Level Alignment at Electrode Interfaces, Nat. Commun. 2014, 5, 4174

[9] van Reenen, S.; Kouijzer, S.; Janssen, R. A. J.; Wienk, M. M.; Kemerink, M., Origin of Work Function Modification by Ionic and Amine-Based Interface Layers. Adv. Mater. Interfaces 2014, 1, 1400189.

[10] Bao, Q.; Liu, X.; Wang, E.; Fang, J.; Gao, F.; Braun, S.; Fahlman, M., Regular Energetics at Conjugated Electrolyte/Electrode Modifier for Organic Electronics and their Implications on Design Rules. Adv. Mater. Interfaces 2015, 2, 1500204.

[11] Bao, Q. Y.; Liu, X. J.; Braun, S.; Gao, F.; Fahlman, M., Energetics at Doped Conjugated Polymer/Electrode Interfaces. Adv. Mater. Interfaces 2015, 2, 1400403. 